\title{
The relationship of C-reactive protein levels and positive culture with quality of life in acute rhinosinusitis
}

\section{Petr Schalek \\ Zuzana Hornáčková \\ Aleš Hahn}

Ear, Nose and Throat Department, 3rd Medical Faculty of Charles University, Prague, Czech Republic
Correspondence: Petr Schalek Ear, Nose and Throat Department, University Hospital Královské Vinohrady Srobárova 50, 10034 Prague, Czech Republic

Tel +420 267162548

Fax +420267I62856

Email schalekp@hotmail.com
This article was published in the following Dove Press journal:

Patient Preference and Adherence

27 January 2015

Number of times this article has been viewed

Background: Acute rhinosinusitis (ARS) has been shown to significantly reduce patient quality of life (QoL). While the QoL in patients with chronic rhinosinusitis has been the subject of intensive research over the last decade, studies measuring the impact of ARS on patient QoL have remained relatively scarce. The aim of this study was to determine the relationship between the QoL and parameters suggestive of acute bacterial rhinosinusitis (C-reactive protein [CRP] levels and positive culture) and to see if measurement of the QoL could be used as an indicator for antibiotic treatment in ARS.

Methods: Eighty patients with ARS were enrolled in the study. A novel QoL instrument for patients with ARS, called Measurement of Acute Rhinosinusitis (MARS) questionnaire, was given to patients at the time of diagnosis. We assessed patient QoL, obtained endoscopically guided cultures from the middle meatus, and measured levels of CRP. The relationship between QoL MARS scores (QoL-Mscores) and CRP was determined using a correlation coefficient. To compare QoL-Mscores, relative to culture-positive and culture-negative patients, the Student's $t$-test was used.

Results: No correlation between the QoL, assessed using the MARS questionnaire, and positive middle meatus culture was demonstrated $(P=0.332)$. A weak correlation was found between QoL-Mscores and CRP values, with a correlation coefficient of 0.221 and $P=0.0498$.

Conclusion: No correlation between the QoL in ARS patients and positive culture was found in this study. The clinical significance of the correlation between QoL-Mscores and CRP values in the antibiotic decision making process needs further research.

Keywords: endoscopy, quality of life, questionnaires, anti-bacterial agents

\section{Introduction}

Upper respiratory infections, including rhinosinusitis, are one of the most common reasons for physician visits. Sinusitis accounts for 12-17 million annual visits to physicians in the United States and for $12 \%$ of antibiotic prescriptions in adults. ${ }^{1,2}$ Acute rhinosinusitis (ARS) significantly reduces patient quality of life (QoL), which in combination with specific symptoms brings the patient to the doctor. While the QoL in patients with chronic rhinosinusitis (CRS) has been the subject of intensive research over the last decade, studies measuring the impact of ARS on patient QoL have remained relatively scarce. ${ }^{3}$

Currently a diagnosis of ARS is based on the assessment of the nature, duration, and severity of symptoms. ${ }^{3}$ The results of objective examinations, relative to ARS, are often of limited value. ${ }^{4}$ Moreover, it is well known that the results of sinus imaging and rhinoendoscopy do not always correlate with patient-reported symptoms of 
rhinosinusitis. ${ }^{5,6}$ Additionally, bacteriology and radiology are not recommended as routine diagnostic methods for making a clinical diagnosis of bacterial ARS, nor are they recommended for guiding treatment. ${ }^{7}$ Therefore, patient-reported outcome measurements represent the best approximation of the burden of the disease on patients.

Most cases of ARS resolve without antibiotic treatment and antibiotics should be reserved for patients with a severe course of the disease. ${ }^{3}$ Despite the fact that antibiotics are almost universally not recommended for treatment of mild, moderate, and uncomplicated ARS, data show that overuse of antibiotics remains an alarming problem. ${ }^{3}$ Although only $0.5 \%-2.0 \%$ of patients develop bacterial rhinosinusitis secondary to a viral infection, ${ }^{3} 50 \%-80 \%$ of patients are treated with antibiotics. ${ }^{7,8}$ It is obvious that no QoL-questionnaire can distinguish between viral and bacterial rhinosinusitis. However, a well-designed questionnaire could identify patients with severe symptoms and significantly reduced QoL; in other words, it might identify patients who could benefit from empirical antibiotic treatment. ${ }^{4}$

The aim of this study was to determine if there was a relationship between patient QoL and parameters suggestive of acute bacterial rhinosinusitis (ie, C-reactive protein [CRP] levels and a positive culture obtained from the middle meatus) and further to determine if QoL measurements could be used as a potential indicator for antibiotic treatment in ARS patients.

A proven correlation between the above-mentioned parameters and QoL would help the physician to better triage patients into immediate, delayed (wait-and-see approach) or no antibiotic treatment.

\section{Patients and methods Patients}

Eighty patients with ARS were enrolled in the study. The diagnosis of ARS was established using the European position paper on rhinosinusitis and nasal polyps 2012 criteria (acute onset of typical symptoms that include nasal blockage, discharge, facial pain and pressure, and reduction of smell $)^{3}$ and confirmed using rhinoendoscopy. Patients were recruited from two ear, nose, and throat offices and the outpatient department of the Royal Vinohrady University Teaching Hospital, Prague, Czech Republic. Enrollees included 44 women and 36 men with a mean age of 39.4 years (18-71 years). All study participants signed an informed consent and the study was approved by the Hospital Ethics Committee (2012 NT/13501-4).

\section{QoL measurement}

A novel QoL instrument for patients with ARS, called Measurement of Acute Rhinosinusitis (MARS) questionnaire, was given to patients at the time of diagnosis. The MARS questionnaire was developed and validated especially for ARS patients and contains 13 items that are evaluated by patients using the Likert scale with four steps $0-3$ ( 0 - no problem, 1 - mild problem, 2 - moderate problem, 3 - severe problem). The range of scores of MARS questionnaire is from 0 (the best QoL) to 39 (the worst possible). Eight items focused on ARS symptoms (nasal obstruction, serous discharge, smell disorders, facial pain or pressure, tenderness over the sinuses, thick or discolored discharge, postnasal discharge, and headache), while five items assessed the social and emotional impact of ARS (fatigue, impaired sleep, reduced concentration during daily activities, irritability, and negatively impacted moods). ${ }^{9}$

\section{Microbiological examination and CRP levels' measurement}

In addition to the QoL measurement, endoscopically guided cultures from the middle meatus and CRP levels determined using a QuikRead ${ }^{\mathrm{TM}}$ device (Orion Diagnostica, Espoo, Finland) were obtained from each study participant. A metaanalysis by Benninger et al confirmed correlation between microbiological samples from the middle meatus and samples from the maxillary sinus. ${ }^{10}$

The swabs from the middle nasal meatus were provided by transport tampone Amies. The specimens were inoculated on Columbia blood agar (Oxoid Ltd., Basingstoke, UK) for cultivation of Staphylococcus aureus, Streptococcus pneumoniae, and Moraxella catarrhalis. The standard media used for growth of Haemophilus influenzae were Levinthal's agar and Chocolate haemophilus selective agar (Oxoid Ltd.). Identification was based on the conventional microbiological methods, and by MALDI-TOF mass spectrometry (Microflex; Bruker Corporation, Billerica, MA, USA).

\section{Statistical analysis}

The relationship between QoL-Mscores (QoL MARS scores) and CRP levels was assessed using a correlation coefficient. The Student's $t$-test was used to compare QoL-Mscores from culture- positive and culture-negative patients. $P$-values $<0.05$ were considered statistically significant. Statistical analysis was performed using Statgraphics software (StatPoint Technologies Inc, Warrenton, VA, USA).

\section{Results}

Positive middle nasal meatus cultures were observed in 24 (30\%) patients while the remaining 56 patients were culture negative. The most common bacteria cultured was $S$. pneumoniae (12 patients), followed by H. influenzae, S. aureus, and M. catarrhalis (six, four, and two patients, respectively). 
The average QoL-Mscores for culture-positive ( $\bar{x}$ MARS $=21.4$ ) (confidence interval 95\% 19.29; 23.54) and culture-negative patients ( $\bar{x}$ MARS $=20.1$ ) (confidence interval 95\% 18.46; 21.78 ) were compared using the Student's $t$-test $(P=0.332)$. In conclusion, no correlation between QoL scores, assessed using the MARS questionnaire, and a positive middle meatus culture was found in this study.

CRP values ranged from 0 to $101 \mathrm{mg} / \mathrm{L},(\bar{x}=23.70$; standard deviation \pm 24.74$)$. The distribution of individual CRP levels was $0-20 \mathrm{mg} / \mathrm{L}$ in 46 patients (57.50\%), 21-100 mg/L in 33 patients $(21.25 \%)$ and in one patient the CRP value was greater than $100 \mathrm{mg} / \mathrm{L}(1.25 \%)$. The correlation coefficient was $r=0.221$ ( $P=0.0498)$. Therefore, there was a significant correlation between QoL-Mscores and CRP values. However, the correlation coefficient and corresponding $P$-value suggest, from a statistical point of view, a rather weak and probably clinically insignificant correlation (Figure 1).

\section{Discussion}

Although the symptoms of ARS are usually transient and recoveries are usually spontaneous, the effect on patient QoL can still be significant. Furthermore, diagnosis and evaluation of therapeutic effects are based on symptomatic changes reported by patients. In his systematic review from 2003, Linder et al stated that the lack of a valid reliable QoL instrument for ARS limited treatment recommendations. ${ }^{4}$ It has been more than 10 years since publication of Linder et al's study and it should be noted that that there has been little movement toward the use of validated QoL instruments for ARS.

With regard to CRS, the relationship between patient QoL and other parameters, has been the subject of intensive research for many years; however, the same cannot be said for ARS, which has only recently become of interest.
So far, we have failed to find any studies that attempted to establish a relationship between patient QoL and bacterial forms of ARS.

The clinical features of viral or bacterial ARS are similar and there are no clinical findings (including character of discharge) that can predict whether a particular case of ARS is of bacterial origin. ${ }^{11}$ The gold standard for assessing the etiology of ARS is a sinus puncture and bacterial culture. However, this invasive procedure is seldom used in primary care. ${ }^{12}$

The most commonly reported bacterial pathogens in ARS are $S$. pneumoniae, $H$. influenzae, $M$. catarrhalis, and $S$. aureus. ${ }^{3,13}$ Even though our results agree and support the data presented in the literature, we found no correlation between a positive culture and patient QoL. Our results suggest that QoL measurements have minimal predictive value for finding a positive culture. But there is limited evidence, in the literature, that a combination of clinical factors (eg, unilateral severe pain, discolored discharge, prolonged illness, or double sickening) is indicative of a more serious disease or constitutes grounds for consideration of antibiotic therapy. ${ }^{7}$

Instant, in office, tests for determination of CRP have been found to be useful indicators of bacterial rhinosinusitis in primary care settings. ${ }^{14,15} \mathrm{CRP}$ levels have been shown to correlate with changes in computed tomography (CT) images and elevated CRP levels have also been shown to be a predictor of positive cultures. ${ }^{10,16}$ Furthermore, CRP determination may be a useful tool for reducing antibiotic overuse in ARS. ${ }^{11,16}$ However, further studies are needed before this test can be recommended as a routine part of the ARS diagnostic protocol that can be used as an indicator for antibiotic treatment.

Hirshoren et al assessed symptom severity, QoL, CT, and CRP levels in patients with severe courses of ARS in an effort to identify patients at risk of impending complications. Thirty-two patients were prospectively enrolled in the study

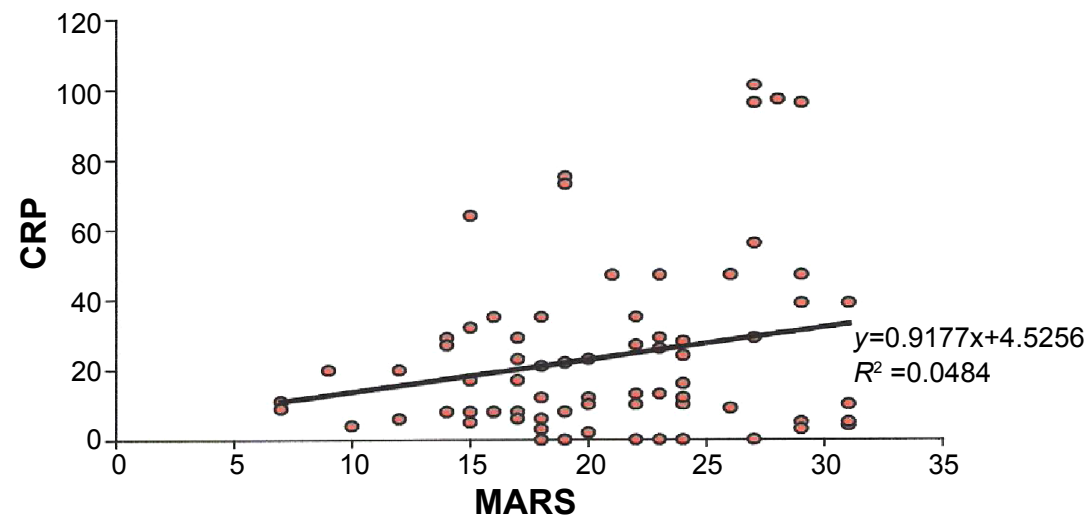

Figure I Correlation between CRP levels and MARS values.

Notes: A weak and probably clinically insignificant correlation was demonstrated (correlation coefficient $r=0.22 \mathrm{I}$ and $P=0.0498$ ). Variations in $C R P$ values associated with specific QoL-Mscores were considerable.

Abbreviations: CRP, C-reactive protein; MARS, Measurement of Acute Rhinosinusitis; QoL, quality of life; Mscores, MARS scores. 
and significant correlations were found between CRP levels, disease severity CT scores, and QoL scores. No complications or surgical interventions were noted in patients with low CRP levels. As with CRS, no correlations between QoL scores and CT scores were found in the study; although, the authors of the study found a strong correlation between high levels of CRP and lower QoL scores $(P=0.0004) .{ }^{17}$

Even though our study found only a weak correlation $(P=0.0498)$ between CRP and QoL scores, the question of its clinical significance remains open. Figure 1 clearly shows that variations in CRP values associated with specific QoLMscores were considerable (for example, a MARS score of 27 was associated with CRP values that range from 0 to 101).

Previous diagnostic research and available evidence regarding CRP being related to respiratory tract infections has recommended a CRP cutoff for antibiotic prescriptions. Antibiotics should not be prescribed when CRP levels are less than $20 \mathrm{mg} / \mathrm{L}$, antibiotics should be prescribed immediately when CRP levels are greater than $100 \mathrm{mg} / \mathrm{L}$ and a wait-andsee approach should be considered for CRP levels between 20 and $99 \mathrm{mg} / \mathrm{L} .^{16,18-20}$

Looking at Figure 1, a CRP value of $20 \mathrm{mg} / \mathrm{L}$ corresponds with a QoL-Mscores of 16 points; while clearly an intriguing correspondence, at this point we can only say that QoL measurements in ARS are useful in evaluation of disease severity and can serve as the primary outcome when evaluating the efficacy of treatment. However, its role in reducing the overuse of antibiotics in the treatment of ARS still needs further evaluation.

Future studies should use standardized methods for making an ARS diagnosis and assessing severity of ARS symptoms. Standardization would facilitate determining if a particular subgroup of signs or symptoms can reliably predict bacterial rhinosinusitis. ${ }^{11}$ Such an assessment would likely be considered incomplete if it did not include QoL measurements. Further studies should also focus on ARS patient follow-up to assess the value of delayed antibiotic treatment relative to QoL measurements.

If QoL measurements are determined to be effective, the advantages of patient QoL assessments become obvious, ie, they are quick, easy, non-invasive, and inexpensive.

\section{Conclusion}

No correlation between the QoL of ARS patients and positive bacterial cultures was found in this study. However, a weak correlation between patient QoL and CRP levels was identified, but its clinical significance and possible contribution to the antibiotic decision making process needs further research.

\section{Disclosure}

The authors have no conflicts of interest to disclose.

\section{References}

1. Gonzales R, Steiner JF, Sande MA. Antibiotic prescribing for adults with colds, upper respiratory tract infections, and bronchitis by ambulatory care physicians. JAMA. 1997;278(11):901-904.

2. Schappert SM. Ambulatory care visits to physician offices, hospital outpatients departments and emergency departments: United States 1996. National Centre for Health Statistics. Vital Health Statistics. 1998;134:1-37.

3. Fokkens WJ, Lund VJ, Mullol, et al. European position paper on rhinosinusitis and nasal polyp. Rhinology Suppl. 2012;23(3):1-298.

4. Linder JA, Singer DE, van der Ancker M, Atlas SJ. Measures of Healthrelated Quality of Life for adults with Acute Sinusitis. J Gen Inter Med. 2003;18(5):390-401.

5. Hopkins C, Browne JP, Slack R, Lund V, Brown P. The Lund-Mackay staging system for chronic rhinosinusitis: how is it used and what does it predict? Otolaryngol Head Neck Surg. 2007;137(4):551-561.

6. Tomassen P, Newson RB, Hoffmans R, et al. Reliability of EP3OS symptom criteria and nasal endoscopy in the assessment of chronic rhinosinusitis-a GA(2)LEN study. Allergy. 2011;66(4):556-561.

7. Fokkens WJ, Hofmans R, Thomas M. Avoid prescribing antibiotics in acute rhinosinusitis. BMJ. 2014;349:g5703.

8. Hansen JG. Acute rhinosinusitis (ARS). Diagnosis and treatment of adults in general practice. Dan Med J. 2014;61(2):B4801.

9. Hornáčková Z, Ježek Z, Kastner J, et al. The MARS questionnaire: Quality of life survey for acute rhinosinusitis. B-ENT. 2014;10:209-213.

10. Benninger MS, Payne SC, Ferguson BJ, Hadley JA, Ahmad N. Endoscopically directed middle meatal cultures versus maxillary sinus taps in acute bacterial maxillary sinusitis: a meta-analysis. Otolaryngol Head Neck Surg. 2006;134(1):3-9.

11. Shintani Smith S, Ference EH, Evans CT, Tan BK, Kern RC, Chandra RK. The prevalence of bacterial infection in acute rhinosinusitis: A Systematic review and meta-analysis. Laryngoscope. Epub 2014 Apr 9.

12. Payne SC, Benninger MS. Staphylococcus aureus is a major pathogen in acute bacterial rhinosinusitis: a meta-analysis. Clin Infect Dis. 2007; 45(10):121-127.

13. Cals JW, Schot MJ, de Jong SA, Dinant GJ, Hopstaken RM. Point-ofcare C-reactive protein testing and antibiotic prescribing for respiratory tract infections: A randomized controlled trial. Ann Fam Med. 2010;8(2):124-133.

14. Llor C, Bjerrum L, Arranz J, et al. C-reactive protein testing in patients with acute rhinosinusitis leads to a reduction in antibiotic use. Fam Pract. 2012;29(6):653-658.

15. Hansen JG, Schmidt H, Rosborg J, Lund E. Predicting acute maxillary sinusitis in a general practice population. BMJ. 1995;311(6999):233-236.

16. Savolainen S, Jousimies-Somer H, Karjalainen J, Ylikoski J. Do simple laboratory test help in etiologic diagnosis in acute maxillary sinusitis? Acta Otolaryngol Suppl. 1997;529:144-147.

17. Hirshoren N, Hirschenbain A, Eliashar R. Risk stratification of severe acute rhinosinusitis unresponsive to oral antibiotics. Acta Otolaryngol. 2010;130(9):1065-1069.

18. Hopstaken RM, Muris JW, Knottnerus JA, Kester AD, Rinkens PE, Dinant GJ. Contributions of symptoms, signs, erythrocyte sedimentation rate and C-reactive protein to diagnosis of pneumonia in acute lower respiratory tract infections. Br J Gen Pract. 2003;53(490):358-364.

19. van der Meer V, Neven AK, ven der Broek PJ, Assendelft WJ. Diagnostic value of C-reactive protein in infections of the lower respiratory tract: systematic review. BMJ. 2005;331(7507):26-29.

20. Bjerrum L, Gahrn-Hansen B, Munck AP. C-reactive protein measurement in general practice may lead to lower antibiotic prescribing for sinusitis. Br J Gen Pract. 2004;54(506):659-662. 
Patient Preference and Adherence

Dovepress

\section{Publish your work in this journal}

Patient Preference and Adherence is an international, peer-reviewed, open access journal that focuses on the growing importance of patient preference and adherence throughout the therapeutic continuum. Patient satisfaction, acceptability, quality of life, compliance, persistence and their role in developing new therapeutic modalities and compounds to optimize

Submit your manuscript here: http://www.dovepress.com/patient-preference-and-adherence-journa clinical outcomes for existing disease states are major areas of interest for the journal. This journal has been accepted for indexing on PubMed Central The manuscript management system is completely online and includes a very quick and fair peer-review system, which is all easy to use. Visit http://www. dovepress.com/testimonials.php to read real quotes from published authors. 